\title{
Double-head lipopeptide surfactants as potential antimicrobial agents
}

Izabela Małuch, Oktawian Stachurski, Paulina Kosikowska-Adamus, Dariusz Wyrzykowski, Adam Prahl, Emilia Sikorska Faculty of Chemistry, University of Gdásk, Poland

https://doi.org/10.17952/35EPS.2018.153

\section{Introduction}

The amphipathicity and total positive charge of short poly-L-lysine lipopeptides make them an interesting group of antimicrobial compounds. Due to their surfactant-like structure they can also exhibit surface-active properties.[1-5] A group of compounds of our particular interest are double-head lipopeptides. Here in, we present the study on the novel non-symmetric lipopeptide surfactants designed by the lipidation of the side chain of L-lysine or its shorter homologs located in the 2 nd position in the peptide sequence.
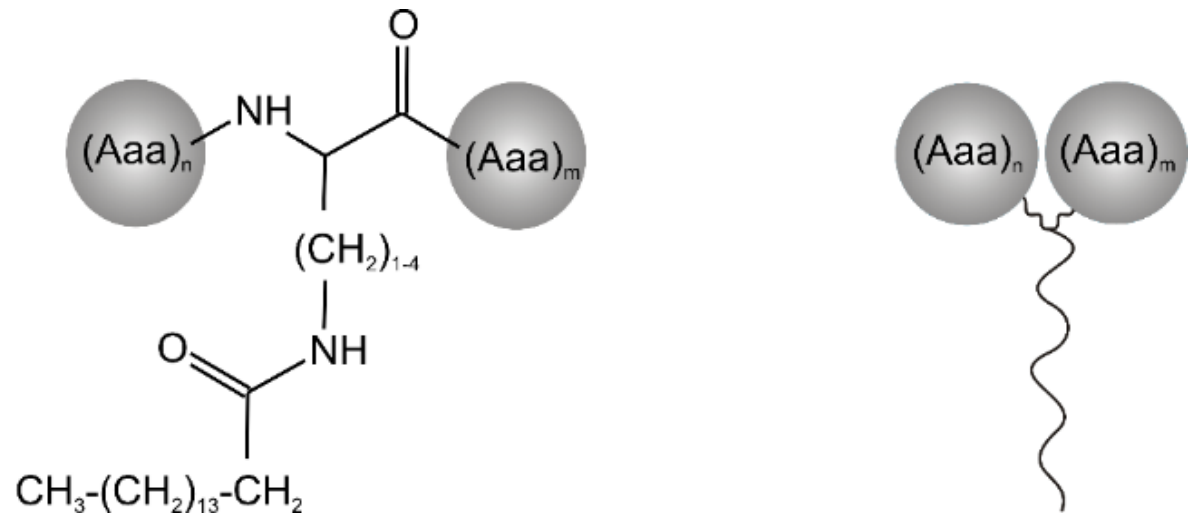

Figure 1: Double-head lipopeptide and schematic representation of the double-head surfactant.

\section{Materials and Methods}

Lipopeptides were prepared by the SPPS method (TentaGel S RAM resin) according to the Fmoc-strategy using automatic peptide synthesizer Sym phony (Protein Technologies). After cleavage from the resin, crude peptides were purified by means of RP-HPLC. Final purity of all synthesized compounds was verified using analytical RP-HPLC system and MALDI-TOF MS was used to confirm their identity. All the lipopeptides were tested for antimicrobial activity. The minimal inhibitory concentration (MIC) values were determined according to standard microdilution technique using 96-well plates. Synthesized lipopeptides were studied for their antimicrobial activity against two bacterial strains (Escherichia coli PCM 2057, Staphylococcus aureus PCM 2054) and one fungal strain (Candida albicans PCM 2566). Mueller-Hintonbroth was used as the working medium for the experiments with bacteria, while RPMI-1640 buffered with MOPS was utilized for $C$. albicans. The effect of the tested compounds upon selected microorganisms was evaluated after 24 hours of co-incubation.

The isothermal titration calorimetry (ITC) was used to determine thermodynamic parameters of lipopeptides binding to anionic liposomes selected as a simplified model of bacterial membrane. Finally, the molecular dynamic simulations using a coarse-grained force fields (CG MD) were applied to visualize the self-assembly properties of the compounds.

\section{Results and Discussion}

We have designed and synthesized four non-symmetric double-head poly-L-lysine lipopeptides (Tab. 1). Based on the initial results of MIC values we have proved that the compounds effectively inhibit visible growth of the selected microorganisms in micromolar range of concentrations $(7.8-15.6 \mathrm{M})$. There is no simple correlation between length of the lipidated amino acid side chain in position 2 and antimicrobial activity. Nevertheless, it seems that peptides modified with longer homologs, Lys and Orn, show slightly better antifungal properties. 
Table 1: Sequences of synthesized lipopeptides, their analytical data and antimicrobial activity.

\begin{tabular}{|c|c|c|c|c|c|c|}
\hline \multirow[t]{2}{*}{ Peptide } & \multirow{2}{*}{$\begin{array}{c}t_{\mathrm{R}} \\
{[\mathrm{min}]^{\mathrm{a}}}\end{array}$} & \multirow{2}{*}{$\begin{array}{c}\text { M } \\
{[\mathrm{Da}]^{\mathbf{b}}}\end{array}$} & \multirow{2}{*}{$\begin{array}{c}{[\mathbf{M}+\mathbf{H}]^{+}} \\
{[\mathbf{D a}]^{\mathbf{c}}}\end{array}$} & \multicolumn{3}{|c|}{$\operatorname{MIC}[\mu \mathrm{g} / \mathrm{mL}]^{\mathrm{d}}$} \\
\hline & & & & E.coli & S.aureus & C.albicans \\
\hline Lys-Lys(Palm)-Lys-Lys-Lys-NH & 22.126 & 896.8 & 897.2 & 7.8 & 15.6 & 7.8 \\
\hline Lys-Orn(Palm)-Lys-Lys-Lys-NH & 21.560 & 882.8 & 883.7 & 15.6 & 15.6 & 7.8 \\
\hline Lys-Dab(Palm)-Lys-Lys-Lys-NH ${ }_{2}$ & 20.790 & 868.7 & 869.5 & 7.8 & 7.8 & 15.6 \\
\hline Lys-Dap(Palm)-Lys-Lys-Lys-NH & 19.769 & 854.7 & 855.4 & 15.6 & 15.6 & 15.6 \\
\hline
\end{tabular}

a) Jupiter $4 \mu$ Proteo $90 \AA$, 250 x $4.6 \mathrm{~mm}$; linear gradient: $15 \%-90 \%$ B in A for 30 minutes, flow rate: $1 \mathrm{ml} / \mathrm{min}$; A $-0.1 \%$ TFA/H2O, B $-80 \% \mathrm{ACN} / \mathrm{H} 2 \mathrm{O}$ containing $0.1 \%$ TFA; b) calculated molecular mass; c) mass observed in MALDI-TOF MS analysis; d) Mueller-Hinton broth was used as the working medium for bacterial strains, while in case of fungi, RPMI-1640 growth medium buffered with MOPS (morpholinepropanesulfonic acid) and additionally supplemented with $2 \%$ glucose was used.

The microbiological results are in good agreement with the outcomes of ITC measurements, which confirm the general ability of the synthesized lipopeptides to interact with negatively charged artificial membrane (POPG LUVs). The titration of Lys-Orn(Palm)-Lys-Lys-Lys- $\mathrm{NH}_{2}$ with POPG exhibits only one steep transition in the ITC curves (Fig. 2) and binding constant of $1.39 \pm 0.6 \times 10^{6} \mathrm{M}^{-1}$. The binding process is exothermic $(\Delta \mathrm{H}=-0.7 \pm 0.23 \mathrm{kcal} / \mathrm{mol})$ and stabilized by entropic factor $(|\Delta \mathrm{H}|<|\mathrm{T} \Delta \mathrm{S}|)$. In the case of the remaining peptides, it was found, that the binding process is accompanied by additional factors, such as lipopeptide aggregation, pore formation, micellization of phospholipid bilayer or change in the lipid phase properties during the titration.[6-8]
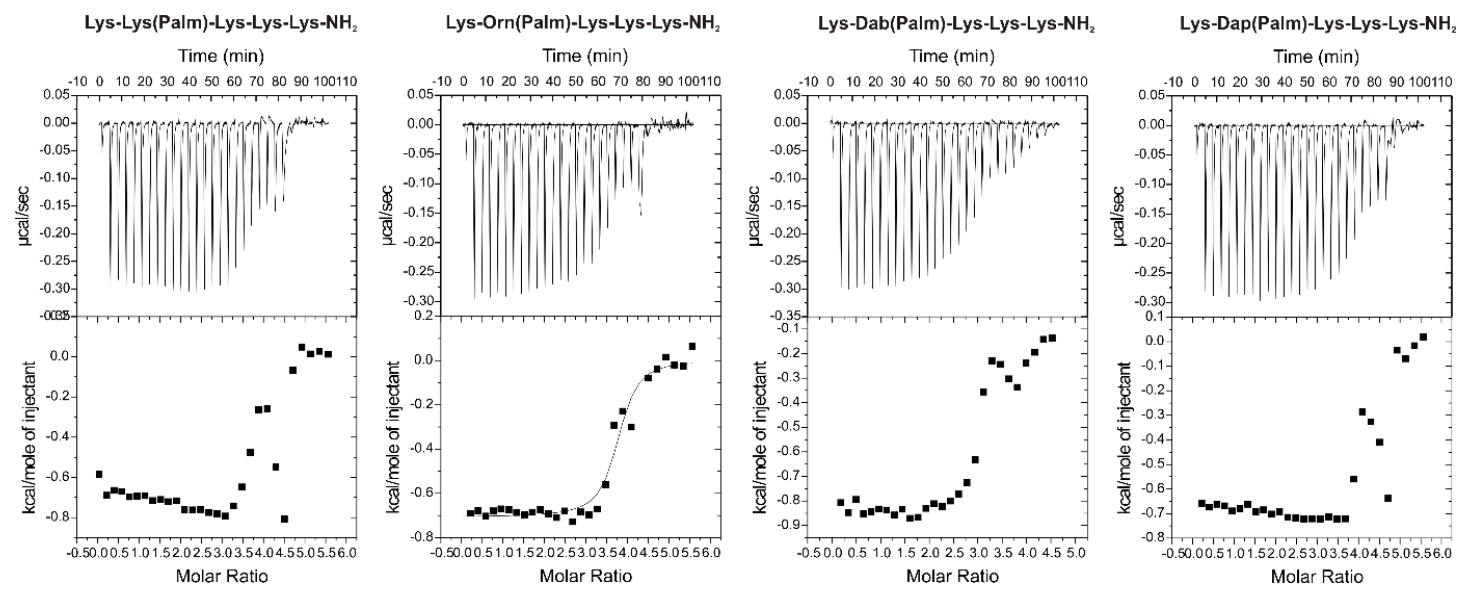

Figure 2: Isothermal titration of 0.05 mM peptide solutions with $1.31 \mathrm{mM}$ POPG LUVs at $298 \mathrm{~K}$. The lower curves represent the heat of reaction (measured by peak integration) as a function of the lipid/peptide molar ratio.

The preliminary results of molecular dynamics simulations indicate the tendency of tested compounds to selfassembly. Figure 3 displays the self-organization of the Lys-Lys(Palm)-Lys-Lys-Lys-NH 2 studied by CG MD simulations.

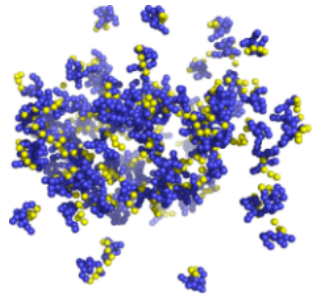

0 ns

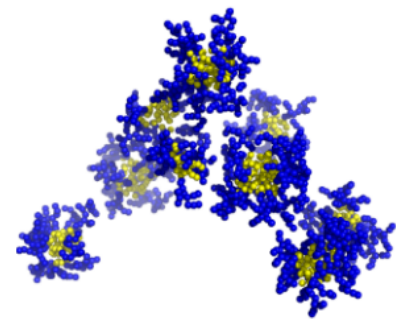

$200 \mathrm{~ns}$

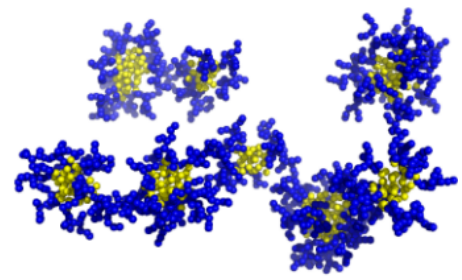

$1000 \mathrm{~ns}$

Figure 3: Snapshots from 1 s CG MD simulations of self-assembly of Lys-Lys(Palm)-Lys-Lys-Lys-NH (water and ions were removed for clarity). Fatty acyl chain is colored yellow.

In summary, the presented type of lipopeptides could serve as a novel model of membrane-targeted antimicrobial compounds. However, in order to establish their potential application additional experiments should be under- 
taken, like evaluation of the toxicity towards eukaryotic cells or determination of critical micelle concentration (CMC) values and size of the formed aggregates. The correlation of biological activity with self-organization would shed light on the plausible mode of action of lipopeptides and open up perspectives for designing analogues with broader antimicrobial and antifungal activities. The results of our studies can contribute to understanding of the self-assembly itself. Compared to the self-assembly of lipids, that of lipopeptides is relatively unexplored.

\section{Acknowledgments}

This work is supported by the National Science Center in Poland, contract No. UMO-2016/21/B/ST5/01375

\section{References}

1. Sikorska, E.; Dawgul, M.; Greber, K.; Iłowska, E.; Pogorzelska, A.; Kamysz, W., BB A Biomembranes 2014, 1838 (10), 2625-2634.

2. Sikorska, E.; Stachurski, O.; Neubauer, D.; Małuch, I.; Wyrzykowski, D.; Bauer, M.; Brzozowski, K.; Kamysz, W., BB A Biomembranes 2018, 1860 (11), 2242-2251.

3. Barchiesi, F.; Giacometti, A.; Cirioni, O.; Arzeni, D.; Silv estri, C.; Kamysz, W.; Abbruzzetti, A.; Riva, A.; Kamysz, E.; Scalise, G., Peptides 2007, 28 (8), 1509-13.

4. Greber, K. E.; Dawgul, M.; Kamysz, W.; Sawicki, W.; Łukasiak, J., Amino acids 2014, 46 (8), 1893-1898.

5. Makovitzki, A.; Baram, J.; Shai, Y., Biochemistry 2008, 47 (40), 10630-10636.

6. Henriksen, J. R.; Andresen, T. L., Biophys J 2011, 101 (1), 100-9.

7. Wenk, M. R.; Seelig, J., Biochemistry 1998, 37 (11), 3909-16.

8. Wenk, M. R.; Seelig, J., J Phys Chem 1997, 101 (26), 5224-5231. 